Research Paper

\title{
The Safety of Chemotherapy for Breast Cancer Patients with Hepatitis C Virus Infection
}

\author{
Yuji Miura ${ }^{\circledR}$, Richard L Theriault ${ }^{2}$, Yoichi Naito ${ }^{1}$, Koichi Suyama $^{1}$, Akihiko Shimomura ${ }^{1}$, Tsuguo Iwatani ${ }^{3}$,
} Daishu Miura ${ }^{3}$, Hidetaka Kawabata ${ }^{3}$, Hiromitsu Kumada ${ }^{4}$, Toshimi Takano ${ }^{1}$

1. Department of Medical Oncology, Toranomon Hospital, Tokyo, Japan.

2. Departments of Breast Medical Oncology, University of Texas M. D. Anderson Cancer Center, Houston, Texas, USA.

3. Department of Breast and Endocrine Surgery, Toranomon Hospital, Tokyo, Japan.

4. Department of Hepatology, Toranomon Hospital, Tokyo, Japan.

$\triangle$ Corresponding author: Dr. Yuji Miura. Department of Medical Oncology, Toranomon Hospital, 2-2-2 Toranomon, Minato-ku, Tokyo, 105-8470, Japan. Tel: 81-3-3588-1111 FAX: 81-3-3582-7068 E-mail: yujmiura@mac.com.

(C) Ivyspring International Publisher. This is an open-access article distributed under the terms of the Creative Commons License (http://creativecommons.org/ licenses/by-nc-nd/3.0/). Reproduction is permitted for personal, noncommercial use, provided that the article is in whole, unmodified, and properly cited.

Received: 2013.03.II; Accepted: 2013.05.23; Published: 20I3.07.24

\begin{abstract}
Background: Hepatitis $\mathrm{C}$ virus (HCV) infection is one of the major causes of chronic liver disease, and more than 880,000 people are estimated to be infected with HCV in Japan. Little information is available on the outcomes of HCV during chemotherapy for solid tumors, and the impact of HCV infection on toxicity of chemotherapy is unknown.

Materials and methods: We performed a retrospective survey of $I, I 10$ patients diagnosed with breast cancer between January 2006 and March 20II at our institution. All patients had been screened for hepatitis $C$ serology at diagnosis of breast cancer. We retrospectively investigated the change in HCV load and the toxicities of chemotherapy, based on review of their medical records.

Results: 23 patients were identified as having a positive test for anti-HCV antibodies. Ten of these patients received chemotherapy. Their median age was 66 years. No patient had decompensated liver disease at baseline. Eight patients received cytotoxic agents with or without trastuzumab, and two patients received trastuzumab alone. Four of eight patients who received cytotoxic chemotherapy developed febrile neutropenia and one developed transaminases elevation. Serum $\mathrm{HCV}$-ribonucleic acid (RNA) level before and after chemotherapy was evaluated in six patients. Median serum HCV-RNA level at baseline and after chemotherapy was 6.5 and 6.7 loglU/ml, respectively.

Conclusion: Chemotherapy for breast cancer patients with HCV infection is feasible, and viral load doesn't change during the chemotherapy.
\end{abstract}

Key words: HCV, HCV-RNA, febrile neutropenia, Child-Pugh criteria, liver cirrhosis, chemotherapy.

\section{Introduction}

Hepatitis C virus (HCV) infection is one of the major causes of chronic liver disease, and more than 880,000 people are estimated to be infected with HCV in Japan (1). The estimated number of HCV carriers increases with age, therefore, carriers aged from 40 to
69 years account for more than $80 \%$ of cases (1). Breast cancer is the most common cancer among Japanese women (2). Furthermore, the age-adjusted breast cancer incidence rate has been increasing since 1975, and the incidence rate of breast cancer is highest in the 
age group of 40-49 years in Japan (2).

Little information is available on the status of $\mathrm{HCV}$ during chemotherapy for solid tumors and the influence of HCV infection on toxicity of chemotherapy is also unknown. Although there are guidelines for management of patients with Hepatitis B virus during chemotherapy, there are no data to support the use of chemotherapy to treat HCV-positive patients with solid tumors $(3,4)$. Some reports have noted the reactivation of HCV in patients with lymphoma who have received rituximab and combination chemotherapy $(5,6)$. However, there are substantial differences in immunosuppressive mechanisms between rituximab-based chemotherapy for hematologic malignancies and conventional chemotherapy for solid tumor, because rituximab, an anti-CD20 antigen, mainly inhibits B-cell function. Therefore, it may not be appropriate to use the same management during chemotherapy for $\mathrm{HCV}$ carrier patients with solid tumors.

The purpose of this study was to evaluate the safety profile and the change in HCV viral load during chemotherapy for $\mathrm{HCV}$-carrier patients with breast cancer.

\section{Materials and methods}

Following data collection and analysis was approved by the Institutional Review Board of Toranomon hospital.

\section{Patients}

We performed a retrospective survey of 1,110 patients diagnosed with breast cancer between January 2006 and March 2011 at our institution. All patients had been screened for hepatitis $C$ serology determined by anti-HCV antibody at diagnosis of breast cancer. Our survey identified 23 patients who were positive for anti-HCV antibodies. The incidence of $\mathrm{HCV}$-positive in breast cancer patients in our institution $(23 / 1,100[2.1 \%])$ is comparable to that in same age range of general women population $(3,221 / 151,501$ [2.1\%]) (1). Ten of these 23 patients received cytotoxic agents and/or trastuzumab. We retrospectively investigated the baseline patient and tumor characteristics, the changes in HCV load, and the toxicities of chemotherapy for these ten patients, based on review of their medical records.

\section{Assessment of breast cancer characteristics}

We collected American Joint Commission on Cancer stage, hormone receptor (HR) status and human epidermal growth factor-2 (HER2)/neu status using immunohistochemistry (IHC) and/or fluorescent in situ hybridization (FISH) at breast cancer di- agnosis. We classified HR-positive as estrogen receptor (ER) positive and/or progesterone receptor (PgR) positive using IHC. The cut point for ER and PgR positivity was an Allred score of 3 (7). HER2 status was defined as positive if an IHC assay demonstrated $3+$ or an IHC score of $2+$ with FISH demonstrated a gene copy ratio of HER2: CEP17 more than 2.2 (8).

\section{Assessment of HCV infection status}

The presence of anti-HCV antibodies was detected using chemiluminescence enzyme immunoassay (Lumipulse Ortho HCV antigen, Ortho-Clinical Diagnostics, Tokyo, Japan) at breast cancer diagnosis. HCV ribonucleic acid (RNA) in serum was detected by a TaqMan Real-Time polymerase chain reaction (PCR) assay (SRL, Tokyo, Japan). In 6 patients, serum HCV-RNA was evaluated before the initiation of chemotherapy and within two months after completing chemotherapy.

\section{Assessment of liver function}

Liver cirrhosis was assessed by clinical criteria (Child-Pugh criteria) at baseline. Data of aspartate aminotransferase (AST), alanine aminotransferase (ALT) and total bilirubin at baseline, during chemotherapy and three months after completion chemotherapy were collected and assessed by Common Terminology Criteria for Adverse Events (CTCAE) version 4.0.

\section{Assessment of other toxicities}

White blood cell count, neutrophil count, platelet count at baseline and during chemotherapy were collected and assessed by CTCAE version 4.0. Events of febrile neutropenia and the use of growth factor support during chemotherapy were collected.

\section{Results}

\section{Patients' characteristics}

Ten of $23 \mathrm{HCV}$-positive patients with breast cancer received cytotoxic agents and/or trastuzumab. Among the remaining 13 patients, ten received only endocrine therapy, two had ductal carcinoma in situ and one was lost to follow-up. The reasons for receiving only hormone therapy among the ten patients included the following; five were low risk of recurrence, three were elderly, age over 75 , and two had decompensated liver function.

Patients and tumor characteristics are shown in Table 1 . The median age at receiving chemotherapy was 66 (range 55-77). Most patients (80\%) had stage II or III disease. Tumor histology of all patients was invasive ductal carcinoma. Tumors were classified on the bases of estrogen/progesterone receptor and 
HER2 status. Three tumors were HR-positive/ HER2-positive, one was HR-positive/HER2-negative, three were HR-negative/HER2-positive, and three tumors were HR-negative/ HER2-negative, respectively. Three patients had a diagnosis of liver cirrhosis. One patient was classified Child B, the remaining two patients were Child A. No patient had decompensated liver disease at baseline.

\section{Treatment course and safety}

Table 2 shows the chemotherapy regimens and their associated documented toxicities. Among the 10 patients who received chemotherapy, three patients received an anthracycline-based regimen, two received anthracycline followed by taxane, two received trastuzumab, two received combination therapy with docetaxel, cyclophosphamide and trastuzumab, and one received gemcitabine.

All patients other than the two who received trastuzumab used dexamethasone as antiemetic prophylaxis. The dose of dexamethasone followed American Society of Clinical Oncology Clinical Prac- tice Guidelines $(9,10)$.

Six $(75 \%)$ of eight patients who received cytotoxic chemotherapy developed grade 4 neutropenia and four $(50 \%)$ developed febrile neutropenia. Transaminases elevated in one (13\%) of eight patients who received cytotoxic chemotherapy. This patient had Child A liver cirrhosis and transaminase elevation at baseline. Four patients received granulocyte colony-stimulating factor (G-CSF). All received the non-pegylated formulation G-CSF. Three patients received G-CSF as a therapeutic use for neutropenia without fever. Two of these patients developed febrile neutropenia. One patient who received G-CSF as a secondary prophylaxis did not develop febrile neutropenia during G-CSF support. No patients received prophylactic antibiotics.

\section{HCV-RNA status}

Table 3 showed the change in HCV-RNA in six patients. The median HCV-RNA before the initiation of chemotherapy was $6.5 \mathrm{log} \mathrm{IU} / \mathrm{ml}$ and after completing chemotherapy was $6.7 \log \mathrm{IU} / \mathrm{ml}$.

Table I. Patients' characteristics.

\begin{tabular}{llllllll}
\hline Case & Age & Stage & Histology & Hormone receptor & HER2 status & LC & Prior HCV therapy \\
\hline 1 & 63 & IIA & IDC & negative & negative & negative & positive \\
2 & 50 & IIA & IDC & positive & negative & negative & negative \\
3 & 50 & IIIA & IDC & positive & negative & negative & negative \\
4 & 61 & IIB & IDC & negative & positive & negative & negative \\
5 & 71 & IIA & IDC & negative & positive & negative & positive \\
6 & 64 & IIA & IDC & positive & positive & positive & negative \\
7 & 67 & IV & IDC & negative & negative & positive & positive \\
8 & 69 & IIA & IDC & negative & positive & negative & negative \\
9 & 60 & IIA & IDC & negative & negative & negative & negative \\
10 & 74 & IV & IDC & positive & negative & positive & negative
\end{tabular}

LC: liver cirrhosis, HCV: hepatitis C virus, IDC: invasive ductal carcinoma.

Table 2. Toxicities

\begin{tabular}{|c|c|c|c|c|c|c|c|c|c|}
\hline Case & $\begin{array}{l}\text { Chemotherapy } \\
\text { regimen }\end{array}$ & $\begin{array}{l}\text { Transaminase } \\
\text { baseline }\end{array}$ & $\begin{array}{l}\text { Transaminase } \\
\text { increase (grade) }\end{array}$ & $\begin{array}{l}\text { WBC/Plt base- } \\
\text { line }\end{array}$ & $\begin{array}{l}\text { Neutropenia } \\
\text { (grade) }\end{array}$ & $\begin{array}{l}\text { Thrombocytopenia } \\
\text { (grade) }\end{array}$ & G-CSF & $\mathrm{FN}$ & $\begin{array}{l}\text { dose reduc- } \\
\text { tion }(\%)\end{array}$ \\
\hline 1 & $\mathrm{EC}$ & increase & 0 & normal & 4 & 3 & positive & positive & 0 \\
\hline 2 & $\mathrm{EC}$ & normal & 0 & normal & 0 & 0 & negative & negative & 0 \\
\hline \multirow[t]{2}{*}{3} & $\mathrm{EC}$ & normal & 0 & normal & 4 & 1 & positive & negative & 0 \\
\hline & DTX & normal & 0 & normal & 4 & 0 & positive & positive & 75 \\
\hline 4 & $\mathrm{TCH}$ & normal & 0 & normal & 4 & 1 & positive & negative & 0 \\
\hline 5 & $\mathrm{H}$ & normal & 0 & normal & 0 & 0 & negative & negative & 0 \\
\hline 6 & $\mathrm{H}$ & normal & 0 & decrease & 0 & 0 & negative & negative & 0 \\
\hline 7 & EC & increase & 1 & decrease & 4 & 3 & negative & positive & 75 \\
\hline 8 & $\mathrm{TCH}$ & normal & 0 & normal & 3 & 0 & negative & negative & 0 \\
\hline \multirow[t]{2}{*}{9} & $\mathrm{EC}$ & normal & 0 & normal & 4 & 3 & positive & positive & 0 \\
\hline & wPTX & normal & 0 & decrease & 4 & 2 & positive & negative & 0 \\
\hline 10 & G & increase & 0 & decrease & 4 & 3 & negative & negative & 75 \\
\hline
\end{tabular}


Table 3. HCV viral load.

\begin{tabular}{lll}
\hline Case & HCV-RNA baseline & $\begin{array}{l}\text { HCV-RNA after chem- } \\
\text { otherapy }\end{array}$ \\
\hline 1 & NA & NA \\
2 & 6.3 & 6.9 \\
3 & 6.7 & 6.7 \\
4 & NA & NA \\
5 & 6.2 & 6.5 \\
6 & 6.5 & 6 \\
7 & 6.7 & 6.7 \\
8 & 6.5 & 6.9 \\
9 & NA & NA \\
10 & NA & NA
\end{tabular}

\section{Discussion}

The present study demonstrates that chemotherapy for breast cancer patients with HCV infection is feasible, and viral load does not vary during the chemotherapy.

A previous study reported that chemotherapy induced elevation of transaminases in patients with $\mathrm{HCV}$ infection $(5,11)$; however the relationship between increase in HCV-RNA and transaminase elevation is poorly investigated. Morrow et al. showed that nine of $36(25 \%) \mathrm{HCV}$ positive patients who received chemotherapy for breast cancer developed elevated liver enzymes, but their study did not evaluate HCV load (12). Our study demonstrated no clinically meaningful changes in HCV-RNA viral load in breast cancer patients who received cytotoxic chemotherapy and/or trastuzumab. It also showed that only one patient $(13 \%)$ had transaminase elevation during chemotherapy. This patient's HCV-RNA was the same before and after chemotherapy. These findings suggest that the elevation of transaminase in our study might not be related to viral reactivation but direct liver toxicity from cytotoxic agents.

Some studies have suggested that B-cell mediated immunosuppression induced by rituximab results in the elevation of transaminase $(5,6,13)$. Coppola et al. showed that rituximab-based chemotherapy resulted in an increase in HCV-RNA at least $1.5 \mathrm{log}$ $\mathrm{IU} / \mathrm{ml}$ (median 2.2 [range 1.5-2.6]) followed by hepatic flare (defined as ALT elevation of more than five times of upper limit of normal or more than 3.6 time of baseline ALT) among patients with lymphoma (6). However, the mechanism of liver injury in HCV infection is still unclear. Further investigations in the relationship between $\mathrm{HCV}$ load and liver injury are warranted.

Previous studies showed a negative impact of corticosteroids on HCV viral load (14). These studies also demonstrated that cumulative exposure to corticosteroids is associated with higher levels of HCV viremia (14). Meanwhile, our study showed that the exposure to dexamethasone use as an antiemetic might not affect $\mathrm{HCV}$ viral load.

Our study showed that $87.5 \%$ patients who received cytotoxic agents developed grade 3-4 neutropenia. Although the incidence of neutropenia depends on the timing and the frequency of blood tests, it is important to note that febrile neutropenia occurred in $50 \%$ of patients who received cytotoxic chemotherapy in our study. A previous study from MD Anderson Cancer Center showed that eight of 36 $(22 \%)$ developed febrile neutropenia (12). Some hypotheses can be raised concerning the causes of the high incidence of febrile neutropenia in our study. The median age of 66 years in the current study was higher than that of the previous report from MD Anderson Cancer Center (48 years) and the highest incidence age of breast cancer in Japan (40-49 years) (2, 12). These findings suggest that older age might contribute to developing the greater incidence of febrile neutropenia as seen in our study. Previous studies of adjuvant chemotherapy for older patients with breast cancer showed that older patients had greater hematologic toxicity, especially neutropenia and febrile neutropenia compared to younger individuals (15, 16). Other potential causes of the high incidence of febrile neutropenia could be considered. Chronic $\mathrm{HCV}$ infection may lead to an immunocompromised status such as neutrophil or T-cell dysfunction. Neutropenia can be seen in patients with chronic hepatitis $\mathrm{C}$ infection due to cirrhosis and hypersplenism. Although four of ten patients had white blood cell counts decrease (grade 1-2) at baseline in the current study, only one of them developed febrile neutropenia (table 2). Although the relationship between $\mathrm{HCV}$ infection and the high incidence of febrile neutropenia is uncertain, clinicians should be concerned with the risk of high-grade neutropenia and febrile neutropenia in $\mathrm{HCV}$-positive patients who receive cytotoxic agents.

\section{Conclusion}

Chemotherapy for breast cancer patients with $\mathrm{HCV}$ infection is feasible and clinically indicated therapy should not be withheld due to positive $\mathrm{HCV}$ serology. Caution regarding neutropenia/ febrile neutropenia is warranted. Use of growth factors may be considered. However, the present study is too small to draw a definite conclusion on the safety of chemotherapy for solid tumors. Further large-scaled investigations are warranted. 


\section{Abbreviations}

HCV: Hepatitis C virus; RNA: ribonucleic acid; HR: hormone receptor; IHC: immunohistochemistry; HER2: human epidermal growth factor-2; FISH: fluorescent in situ hybridization; PCR: polymerase chain reaction; AST: aspartate aminotransferase; ALT: alanine aminotransferase; CTCAE: Common Terminology Criteria for Adverse Events; G-CSF: granulocyte colony-stimulating factor.

\section{Acknowledgement}

The authors thank Japan TeamOncology Program (J-TOP) for their educational programs.

\section{Ethics Committee Approval}

The data collection and analysis of the current study was approved by the Institutional Review Board of Toranomon hospital.

\section{Competing interests}

Dr. Kumada received grant from MSD K.K., Bristol-Myers Squibb K.K., Dainippon Sumitomo Pharma, and Daiichi Sankyo. All other authors state that they have no conflicts of interest.

\section{References}

1. Tanaka J, Kumagai J, Katayama K, et al. Sex- and age-specific carriers of hepatitis $B$ and $C$ viruses in Japan estimated by the prevalence in the 3,485,648 first-time blood donors during 1995-2000. Intervirology. 2004;47:32-40.

2. Matsuda T, Marugame T, Kamo K, et al. Cancer incidence and incidence rates in Japan in 2006: based on data from 15 population-based cancer registries in the monitoring of cancer incidence in Japan (MCIJ) project. Japanese journal of clinical oncology. 2012;42:139-47.

3. Artz AS, Somerfield MR, Feld JJ, et al. American Society of Clinical Oncology provisional clinical opinion: chronic hepatitis B virus infection screening in patients receiving cytotoxic chemotherapy for treatment of malignant diseases. Journal of clinical oncology : official journal of the American Society of Clinical Oncology. 2010;28:3199-202.

4. Lok AS, McMahon BJ. Chronic hepatitis B: update 2009. Hepatology. 2009:50:661-2.

5. Hsieh CY, Huang HH, Lin CY, et al. Rituximab-induced hepatitis C virus reactivation after spontaneous remission in diffuse large B-cell lymphoma. Journal of clinical oncology : official journal of the American Society of Clinical Oncology. 2008;26:2584-6.

6. Coppola N, Pisaturo M, Guastafierro S, et al. Increased hepatitis C viral load and reactivation of liver disease in HCV RNA-positive patients with onco-haematological disease undergoing chemotherapy. Dig Liver Dis. 2012;44:49-54.

7. Allred DC, Harvey JM, Berardo M, et al. Prognostic and predictive factors in breast cancer by immunohistochemical analysis. Modern pathology : an official journal of the United States and Canadian Academy of Pathology, Inc. 1998;11:155-68.

8. Wolff AC, Hammond ME, Schwartz JN, et al. American Society of Clinical Oncology/College of American Pathologists guideline recommendations for human epidermal growth factor receptor 2 testing in breast cancer. Journal of clinical oncology : official journal of the American Society of Clinical Oncology. 2007;25:118-45.

9. Kris MG, Hesketh PJ, Somerfield MR, et al. American Society of Clinical Oncology guideline for antiemetics in oncology: update 2006. Journal of clinical oncology : official journal of the American Society of Clinical Oncology. 2006;24:2932-47.

10. Basch E, Prestrud AA, Hesketh PJ, et al. Antiemetics: American Society of Clinical Oncology clinical practice guideline update. Journal of clinical oncology : official journal of the American Society of Clinical Oncology. 2011;29:4189-98.

11. Melisko ME, Fox R, Venook A. Reactivation of hepatitis $C$ virus after chemotherapy for colon cancer. Clin Oncol (R Coll Radiol). 2004;16:204-5.

12. Morrow PK, Tarrand JJ, Taylor SH, et al. Effects of chronic hepatitis C infection on the treatment of breast cancer patients. Ann Oncol. 2010;21:1233-6.

13. Nooka A, Shenoy PJ, Sinha $R$, et al. Hepatitis $C$ reactivation in patients who have diffuse large B-cell lymphoma treated with rituximab: a case report and review of literature. Clin Lymphoma Myeloma Leuk. 2011;11:379-84.

14. Charlton M. Hepatitis $C$ infection in liver transplantation. American journal of transplantation : official journal of the American Society of Transplantation and the American Society of Transplant Surgeons. 2001;1:197-203.

15. Muss HB, Berry DA, Cirrincione C, et al. Toxicity of older and younger patients treated with adjuvant chemotherapy for node-positive breast cancer: the Cancer and Leukemia Group B Experience. Journal of clinical oncology : official journal of the American Society of Clinical Oncology. 2007;25:3699-704.

16. Crivellari D, Bonetti M, Castiglione-Gertsch M, et al. Burdens and benefits of adjuvant cyclophosphamide, methotrexate, and fluorouracil and tamoxifen for elderly patients with breast cancer: the International Breast Cancer Study Group Trial VII. Journal of clinical oncology : official journal of the American Society of Clinical Oncology. 2000;18:1412-22. 